\title{
ON THE COMPRESSION OF A SHORT CYLINDER BETWEEN ROUGH END-BLOCKS*
}

\author{
By F. EDELMAN (Brown University)
}

1. In this paper we consider non-uniform elastic compression between parallel endblocks of a cylindrical test specimen with circular cross-section. It is assumed that there is sufficient friction between the end-blocks and the end faces of the specimen to prevent slippage. Thus, points on the end faces of the cylinder undergo only axial displacements.

It is the purpose of this paper to determine the relation between the force $F$ acting on the specimen, the compression $2 a$, and Young's modulus $E$ of the material, assuming Poisson's ratio $\nu$ to be known. If lateral expansion were not obstructed by the friction at the end surfaces, these quantities would be connected by the relation $E=F h / A a$, where $2 h$ denotes the height of the specimen, $b$ its radius and $A$ its cross-sectional area. If there is friction at the end surfaces, a correction must be applied to this simple formula; the derivation of this correction will now be sketched.

The above problem is solved to a good approximation by finding upper and lower bounds for the strain energy of the specimen, using the method of Prager and Synge. ${ }^{1}$

It is advantageous to resolve the original problem, which we shall denote by $P_{0}$, into two simpler problems $P_{c}$ and $P$, where $P_{c}$ is the problem of simple compression and $P$ is that problem which will yield the initial problem $P_{0}$ when superimposed on $P_{c}$.

In the natural cylindrical coordinates, the original problem $P_{0}$ involves the boundary conditions

$$
\begin{array}{ll}
u_{r}(r, \pm h)=0, & u_{z}(r, \pm h)=\mp a, \\
T_{n}(b, z)=0, & T_{\imath}(b, z)=0,
\end{array}
$$

where $u_{r}$ and $u_{z}$ are the displacements in the $r$ and $z$ directions respectively and $T_{n}$, $T_{t}$ are the normal and tangential components of stress transmitted through the surface.

Thus the problem $P$, with which we shall deal from now on, has the boundary conditions

$$
\begin{array}{ll}
u_{r}(r, \pm h)=-\nu a r, & u_{z}(r, \pm h)=0, \\
T_{n}(b, z)=0, & T_{\imath}(b, z)=0 .
\end{array}
$$

The analysis used in the following is quite similar to that given in a previous paper ${ }^{2}$ in which the case of plane strain was considered, except that here we have cylindrical coordinates and different boundary conditions. The similarity, however, permits the procedure and results alone to be indicated here.

2. Since the bar possesses rotational symmetry, the displacements $u_{r}$ and $u_{z}$ are functions of $r$ and $z$ only and not of $\theta$, and there is no circumferential displacement, i.e. $u_{\theta}=\partial u / \partial \theta=0$.

*Received Sept. 22, 1948.

${ }^{1}$ W. Prager and J. L. Synge, Approximations in elasticity based on the concept of function space, Q. Appl. Math. 5, 241-269 (1947).

${ }^{2} \mathrm{H}$. J. Greenberg and R. Truell, On a problem in plane strain, Q. Appl. Math. 6, 53 (1948). 
Hooke's law then has the form

$$
\begin{aligned}
& e_{r r}=\frac{1}{E}\left[E_{r r}-\nu\left(E_{\theta \theta}+E_{z z}\right)\right] \\
& e_{z z}=\frac{1}{E}\left[E_{z z}-\nu\left(E_{r r}+E_{\theta \theta}\right)\right], \\
& e_{\theta \theta}=\frac{1}{E}\left[E_{\theta \theta}-\nu\left(E_{r r}+E_{z z}\right)\right], \\
& e_{r z}=\frac{1+\nu}{E} E_{r z} \\
& e_{r \theta}=e_{z \theta}=0
\end{aligned}
$$

and

$$
\begin{aligned}
& E_{r r}=\frac{E}{(1+\nu)(1-2 \nu)}\left[(1-\nu) e_{r r}+\nu\left(e_{\theta \theta}+e_{z z}\right)\right], \\
& E_{z z}=\frac{E}{(1+\nu)(1-2 \nu)}\left[(1-\nu) e_{z z}+\nu\left(e_{r r}+e_{\theta \theta}\right)\right], \\
& E_{\theta \theta}=\frac{E}{(1+\nu)(1-2 \nu)}\left[(1-\nu) e_{\theta \theta}+\nu\left(e_{r r}+e_{z z}\right)\right], \\
& E_{r z}=\frac{E}{1+\nu} e_{r z}, \quad E_{r \theta}=E_{z \theta}=0 .
\end{aligned}
$$

The strains are defined in terms of the displacements by

$$
\left.\begin{array}{ll}
e_{r r}=\frac{\partial u_{r}}{\partial r}, & e_{z z}=\frac{\partial u_{z}}{\partial z} \\
e_{\theta \theta}=\frac{u_{r}}{r}, & e_{r z}=\frac{1}{2}\left(\frac{\partial u_{r}}{\partial z}+\frac{\partial u_{z}}{\partial r}\right) .
\end{array}\right\}
$$

The equilibrium equations reduce to

$$
\frac{\partial}{\partial r}\left(r E_{r r}\right)+\frac{\partial}{\partial z}\left(r E_{r z}\right)=E_{\theta \theta}, \quad \frac{\partial}{\partial r}\left(r E_{r z}\right)+\frac{\partial}{\partial z}\left(r E_{z z}\right)=0 .
$$

The Eqs. (6) are satisfied automatically if we choose our stresses to be

$$
\begin{array}{ll}
E_{r r}=\frac{\psi}{r}, & E_{z z}=-\frac{1}{r} \frac{\partial \varphi}{\partial r}, \\
E_{\theta \theta}=\frac{\partial \psi}{\partial r}+\frac{\partial^{2} \varphi}{\partial z^{2}}, & E_{r z}=\frac{1}{r} \frac{\partial \varphi}{\partial z},
\end{array}
$$

where $\psi$ and $\varphi$ are two arbitrary functions, called the stress functions.

Following the Prager-Synge notation and development we have the following boundary conditions: 
a) On $\mathbf{S}^{*}$, the completely associated state,

$$
u_{r}^{*}(r, \pm h)=-\nu a r, \quad u_{z}^{*}(r, \pm h)=0 ;
$$

b) On $\mathbf{S}_{p}^{\prime}$, the homogeneous associated states,

$$
{ }_{p} u_{r}^{\prime}(r, \pm h)=0, \quad{ }_{p} u_{z}^{\prime}(r, \pm h)=0 ;
$$

c) On $\mathbf{S}_{a}^{\prime \prime}$, the complimentary states,

$$
{ }_{a} E_{r r}^{\prime \prime}(b, z)=0, \quad{ }_{a} E_{r z}^{\prime \prime}(b, z)=0,
$$

where the stresses ${ }_{a} E_{i j}^{\prime \prime}$ must satisfy the equilibrium conditions (6).

3. If $\mathrm{S}_{0}, \mathrm{~S}_{c}$ and $\mathrm{S}$ are the "natural" states of the problems $P_{0}, P_{c}$ and $P$ respectively, then it can be seen at once that the strain energies can be superimposed by simple addition.

Thus

$$
S_{0}^{2}=S_{c}^{2}+S^{2}
$$

since the boundary conditions of $P_{c}$ and $P$ are such that the scalar product $\mathbf{S}_{c} \cdot \mathbf{S}=$ $\int T_{i}^{c} u_{i} d s$ vanishes. Finding bounds on $S_{0}^{2}$ therefore reduces to finding bounds on $S^{2}$ since $S_{c}^{2}$ is known to be $2 \pi a^{2} E$.

Prager-Synge give the following inequality for $S^{2}$ :

$$
\sum_{a=1}^{n}\left(S^{*} \cdot I_{a}^{\prime \prime}\right)^{2} \leq S^{2} \leq S^{* 2}-\sum_{p=1}^{m}\left(S^{*} \cdot I_{p}^{\prime}\right)^{2}
$$

where $\mathbf{I}_{p}^{\prime}, \mathbf{I}_{a}^{\prime \prime}$ are obtained by orthonormalizing the sequences $\mathbf{S}_{p}^{\prime}, \mathbf{S}_{a}^{\prime \prime}$ respectively. As in footnote 2 , this reduces to

where

$$
\frac{2}{\pi\left(U_{m}+2\right)} \frac{F}{a} \leq E \leq \frac{2}{\pi\left(L_{n}+2\right)} \frac{F}{a}
$$

$$
L_{n}=\frac{1}{\pi a^{2} E} \sum_{a=1}^{n}\left(\mathbf{S}^{*} \cdot \mathbf{I}_{a}^{\prime \prime}\right)^{2}, \quad U_{m}=\frac{1}{\pi a^{2} E}\left[S^{*^{2}}-\sum_{p=1}^{m}\left(\mathbf{S}^{*} \cdot \mathbf{I}_{p}^{\prime}\right)^{2}\right] .
$$

4. Suitable displacement functions, satisfying appropriate boundary conditions and obvious symmetry conditions, are chosen to obtain the states $\mathbf{S}^{*}$ and $\mathbf{S}_{p}^{\prime}$. Stress functions $\varphi$ and $\psi$ are selected for the computation of the states $\mathbf{S}_{a}^{\prime \prime}$. The orthonormalization is carried out using the Gram-Schmidt procedure.

The bounds $U_{m}$ and $L_{n}$ are computed at each iteration and after five steps we obtain the inequality

$$
0.29958 \frac{F}{a} \leq E \leq 0.30371 \frac{F}{a},
$$

where we have now set $b=h=1$ and $\nu=1 / 3$.

Averaging, we have

$$
E=0.3017 \frac{F}{a} .
$$

In terms of the mean stress $\sigma=F / \pi b^{2}$ and the mean strain $\epsilon=a / h$, we obtain

$$
E=0.9477 \frac{\sigma}{\epsilon} .
$$


Let us now perform the test in which the specimen is in a state of compression, such as in problem $P_{0}$, and measure the applied force $F$ and the compression $2 a$. The formula

$$
\bar{E}=\frac{F h}{A a}=\frac{\sigma}{\epsilon}
$$

no longer yields the true value of $E$ since this formula applies exactly only to the test in which there is no friction at the end-blocks, i.e. simple compression. However the true value of $E$ is given by Eq. (16) and the relation between $E$ and $\bar{E}$ is found by comparison of (16) and (17), namely

$$
E=0.9477 \bar{E} .
$$

This gives us the correction factor to be applied to Eq. (17) to yield the true value of Young's modulus $E$. The numerical coefficient of $\bar{E}$ in (18) is in error by less than $0.7 \%$.

The displacements and strains of the completely associated state and the homogeneous associated states are given in Table I below. The stress functions and stresses of the complementary states are shown in Table II.

TABLE I. The Associated States

\begin{tabular}{|c|c|c|c|c|c|c|}
\hline & $u_{r}^{\prime}$ & $u_{z}^{\prime}$ & $e_{r r}^{\prime}$ & $e_{\theta \theta}^{\prime}$ & $e_{z s}^{\prime}$ & $e_{r s}^{\prime}$ \\
\hline $\mathbf{S}_{1}^{\prime}$ & 0 & $-\left(1-z^{2}\right) z$ & 0 & 0 & $-\left(1-3 z^{2}\right)$ & 0 \\
\hline $\mathbf{S}_{2}^{\prime}$ & $r z^{2}\left(1-z^{2}\right)$ & 0 & $z^{2}\left(1-z^{2}\right)$ & $z^{2}\left(1-z^{2}\right)$ & 0 & $r z\left(1-2 z^{2}\right)$ \\
\hline $\mathrm{S}_{3}^{\prime}$ & 0 & $-r^{2}\left(1-z^{2}\right) z$ & 0 & 0 & $-r^{2}\left(1-3 z^{2}\right)$ & $-r z\left(1-z^{2}\right)$ \\
\hline $\mathrm{S}_{4}^{\prime}$ & $r^{2} z^{2}\left(1-z^{2}\right)$ & 0 & $2 r z^{2}\left(1-z^{2}\right)$ & $r z^{2}\left(1-z^{2}\right)$ & 0 & $r^{2} z\left(1-2 z^{2}\right)$ \\
\hline $\mathrm{S}_{5}^{\prime}$ & 0 & $-r\left(1-z^{2}\right) z$ & 0 & 0 & $-r\left(1-3 z^{2}\right)$ & $-\frac{1}{2} z\left(1-z^{2}\right)$ \\
\hline $\mathrm{S}^{*}$ & $-\frac{1}{3} r z^{4}$ & 0 & $-\frac{1}{3} z^{4}$ & $-\frac{1}{3} z^{4}$ & 0 & $-\frac{2}{3} r z^{3}$ \\
\hline
\end{tabular}

TABLE II. The Complementary States.

\begin{tabular}{|c|c|c|c|c|c|c|}
\hline & $\varphi$ & $\psi$ & $E_{r r}^{\prime \prime}$ & $E_{\theta \theta}^{\prime \prime}$ & $E_{z z}^{\prime \prime}$ & $E_{r z}^{\prime \prime}$ \\
\hline $\mathbf{S}_{1}^{\prime \prime}$ & $\frac{1}{2} z^{4} r^{2}\left(1-r^{2}\right)$ & 0 & 0 & $6 z^{2} r^{2}\left(1-r^{2}\right)$ & $z^{4}\left(2 r^{2}-1\right)$ & $2 z^{3} r\left(1-r^{2}\right)$ \\
\hline $\mathbf{S}_{2}^{\prime \prime}$ & 0 & $z^{2} r\left(1-r^{2}\right)$ & $z^{2}\left(1-r^{2}\right)$ & $z^{2}\left(1-3 r^{2}\right)$ & 0 & 0 \\
\hline $\mathbf{S}_{3}^{\prime \prime}$ & $\frac{1}{2} r^{2}$ & 0 & 0 & 0 & -1 & 0 \\
\hline $\mathbf{S}_{4}^{\prime \prime}$ & 0 & $z^{2} r^{2}\left(1-r^{2}\right)$ & $z^{2} r\left(1-r^{2}\right)$ & $2 z^{2} r\left(1-2 r^{2}\right)$ & 0 & 0 \\
\hline $\mathbf{S}_{5}^{\prime \prime}$ & $\frac{1}{3} r^{3}$ & 0 & 0 & 0 & $-r$ & 0 \\
\hline
\end{tabular}

